\title{
The EQUATOR network and website
}

\author{
Derek Richards \\ Centre for Evidence-based Dentistry, Oxford, UK
}

Evidence-Based Dentistry (2007) 8, 117. doi:10.1038/sj.ebd.6400533

The quality, reliability and reporting of research is a subject that is regularly raised in the pages of Evidence-based Dentistry. We have constantly sought to highlight the various guidelines available to guide researchers when reporting their research and, consequently, it is pleasing to be able to bring to readers' attention a new group, the EQUATOR Network (Enhancing the QUAlity and Transparency Of health Research). This is a new quality-improvement initiative that seeks to improve the quality and reliability of the health research literature by promoting transparent and accurate reporting through the use of reporting guidelines.

These guidelines specify the minimum information necessary for a clear account of research methods and findings. Use of reporting guidelines can lead to improved accuracy and clarity of publications and facilitate appraisal of research quality and relevance. It can also improve the efficiency of literature searches and the ability to find information. During the last 10 years, a considerable number of guidelines have been developed for reporting health research. As in areas other than dentistry, although good-quality reporting guidelines exist, they are still not widely used and implemented and their benefits are not, therefore, being fully realised.

The EQUATOR Network aims to remedy this situation and become a recognised global centre that provides resources and training relating to the reporting of health research, and assist in the development, dissemination and implementation of reporting guidelines. The work is being led by Professor Doug Altman, who earlier this year was presented with the Council of Science Editors Annual Award for Meritorious Achievement for his work to improve the standards of reporting of clinical research. Professor Altman is Director of the Centre for Statistics in Medicine at the University of Oxford, UK, and is one of the leading experts in the field of health research methodology, statistics and reporting. He is one of the key movers in the CONSORT (Consolidated Standards of Reporting Trials) initiative.

The EQUATOR Network has an international steering group which, in addition to Professor Altman, includes leading experts in the field of health research methodology, reporting and editorial work. The network plans to develop:

- a web-based resource centre with easy access to reporting guide-

lines and other relevant information;

- support for the development of robust reporting guidelines;

- training courses for authors, editors and peer reviewers, facilitating transparent and accurate reporting and use of reporting guidelines;

- an annual assessment of how journals implement reporting guidelines;

- an annual audit of reporting quality across the health research literature.
The EQUATOR Network has developed from the work of CONSORT and other guideline development groups. It began in March 2006 as a project funded by the UK National Health Service National Knowledge Service with three major objectives: to map the current status of all activities aiming at the preparation and dissemination of guidelines on reporting health research studies; to identify key individuals working in the area; and to establish relationships with potential key stakeholders. The EQUATOR Network held its first international meeting in Oxford in MayJune 2006, which was attended by 27 participants from 10 countries: the formal launch of the network and website is one of its outcomes.

\section{EQUATOR website}

The website (www.equator-network.org) has recently been launched (Fig. 1) and one of its key features is a collection of reporting guidelines that includes:

- CONSORT statement (for reporting randomised controlled trials)

- QUOROM, recently renamed PRISMA (for reporting systematic reviews and meta-analyses of randomised trials)

- STROBE statement (for reporting observational studies in epidemiology)

- STARD statement (for reporting diagnostic accuracy studies)

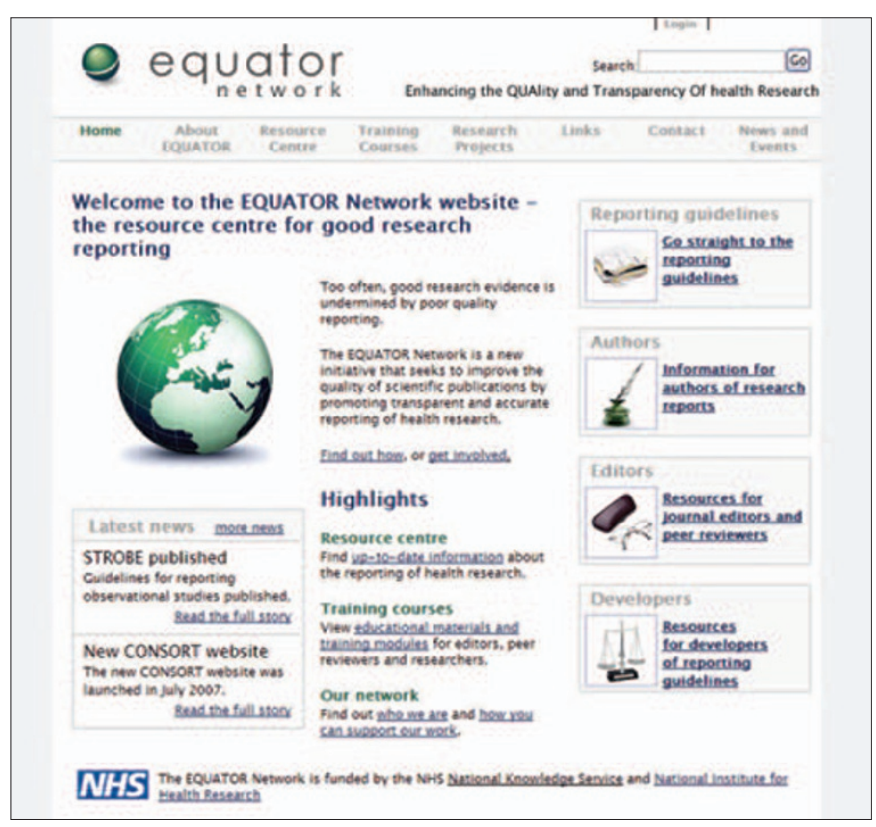

Figure 1. The Equator Network homepage 